\title{
Magnetic characterization of Fe-Al-B amorphous ribbons obtained by the melt spinning technique
}

\author{
J.A. García, J. Carrizo, L. Elbaile, M. Rivas, R.D. Crespo, and J.C. Martínez-García \\ Department of Physics. University of Oviedo, c/ Calvo Sotelo s/n, 33007 Oviedo, Spain
}

\begin{abstract}
The magnetic properties of a new amorphous material obtained by melt spinning technique is presented. The composition of the ribbon is $\mathrm{Fe}_{72} \mathrm{Al}_{14} \mathrm{~B}_{14}$. The bulk hysteresis loops indicates that this ribbon is a soft magnetic material with a magnetic anisotropy along the longitudinal direction of the ribbon and an anisotropy constant of $1860 \mathrm{Jm}^{-3}$. The value of the anisotropy is explained on the basis of the formation of directional order. The saturation magnetization results $1.12 \mathrm{~T}$ and the coercive field $18 \mathrm{Am}^{-1}$. The surface magnetic properties show that the magnetic anisotropy is higher in the wheel-surface than in the air-surface. This result is explained considering the residual stress distribution in this kind of materials.
\end{abstract}

\section{Introduction}

In the last years there has been an intense development in sensor technology for a wide range of applications. In the case of sensors for steering shaft (to be used in cars, ships, etc) the non contact sensors are the most used and among them, the magnetostrictive sensors suit very well due to their contactless sensing capabilities $[1,2]$. For the above mentioned application on steering shaft, ribbons of highly magnetostrictive materials are very convenient due to their shape which allows them to be tightly attached to the shaft. In this field, different magnetic sensors based on amorphous magnetostrictive ribbons have been developed [3,4]. Recently, the production by melt spinning of crystalline ribbons of Fe-Al and Fe-Ga [5,6] with very high magnetostriction has generated a renewed interest in these alloys as potential cores for magnetic sensors. Nevertheless, these ribbons have neither good mechanical properties nor high corrosion resistance. Good mechanical properties of the ribbon used in the sensor are fundamental in order to avoid damage that could produce failures in its working. Moreover, high corrosion resistance is required when the sensor has to work in aggressive mediums as for example maritime environment. In the case of $\mathrm{Fe}-\mathrm{Al}$ alloys, the addition of $\mathrm{B}$ improves their mechanical properties but, it has been shown that rapidly quenched Fe-Al-B alloys containing 20-40 atom per cent aluminium and 5-7 per cent atom boron are extremely brittle [7]. Recently, some of the authors have produced by the melt spinning amorphous ribbons of composition $\mathrm{Fe}_{72} \mathrm{Al}_{14} \mathrm{~B}_{14}$ with excellent mechanical properties [8] and higher corrosion resistance than the Fe-Al alloys [9].
In this work the magnetic characterization of the novel amorphous ribbons $\mathrm{Fe}_{72} \mathrm{Al}_{14} \mathrm{~B}_{14}$ obtained by melt spinning technique is presented.

\section{Experimental procedure}

Alloys ingots of $\mathrm{Fe}_{72} \mathrm{Al}_{14} \mathrm{~B}_{14}$ were prepared by induction melting under vacuum atmosphere. Ribbons of $1 \mathrm{~mm}$ wide and $40 \mu \mathrm{m}$ thick were produced from these ingots by planar flow casting. The melting temperature was $1250^{\circ} \mathrm{C}$, the wheel speed $1.8 \mathrm{~m} / \mathrm{s}$ and the ejection pressure 400 mbar.

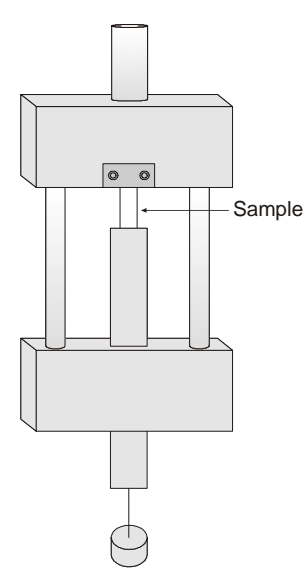

Fig. 1. Schematic representation of the holder for the magnetostriction measurements.

The structural characterization of the ribbon was performed by X-ray spectroscopy. The bulk hysteresis loops were obtained in a hysteresis loop tracer with drift 
correction development in our laboratory [10]. The surface hysteresis loops were obtained by transverse magneto-optical Kerr effect. The light is linearly polarized in the plane of incidence, which is perpendicular to the applied magnetic field. The radiation is directed to the sample at an angle of incidence of $65^{\circ}$.

Magnetostriction was measured using the strain-gauge technique. To avoid any flexion of the ribbon which can induce spurious deformation of the sample, a holder similar to that developed by Takahasi et al. [11] and which is represented schematically in figure 1 has been used.

\section{Results and discussion}

The amorphous state of the ribbon has been confirmed by X-ray diffraction. As can be seen in figure 2 the XRD spectrum of the as-quenched ribbon consists only of a broad halo characteristic of the amorphous phase, with no sharp peaks corresponding to crystalline phases.

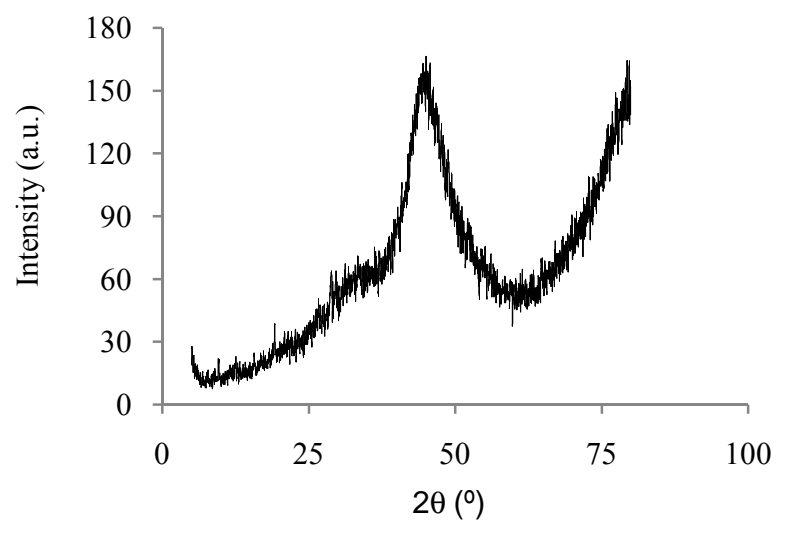

Fig. 2. XRD pattern of the $\mathrm{Fe}_{72} \mathrm{Al}_{14} \mathrm{~B}_{14}$ ribbon.

Figure 3 illustrates the enlarged image of the central part of the bulk hysteresis loop shown in the inset. It indicates clearly that amorphous $\mathrm{Fe}_{72} \mathrm{Al}_{14} \mathrm{~B}_{14}$ is magnetically soft. The saturation magnetization $\mu_{0} M_{s}$ results in $1.12 \mathrm{~T}$ and the coercive field $H_{c}$ is $18 \mathrm{Am}^{-1}$.

From the bulk hysteresis loop and taking into account the relation between the anisotropy field and the saturation magnetization,

$H_{k}=\frac{2 K}{\mu_{0} M_{s}}$

a value for the anisotropy constant $K$ of $1680 \mathrm{Jm}^{-3}$ is obtained. This high value of the anisotropy can be attributed to the directional order due to the formation of $\mathrm{Fe}-\mathrm{Fe}$ pairs. A similar effect has been reported in polycrystalline rapidly quenched $\mathrm{Fe}-\mathrm{Al}$ [5] and $\mathrm{Fe}_{81} \mathrm{Ga}_{19}$ [12].

The longitudinal magnetostriction of the ribbon measured under a transverse magnetic field of $0.9 \mathrm{~T}$ results in $\lambda_{s}=24 \mathrm{ppm}$.

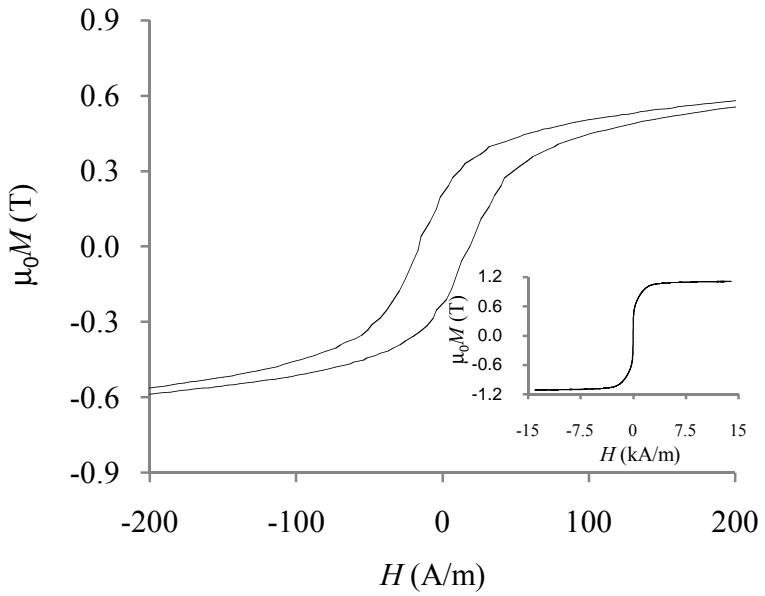

Fig. 3. Bulk hysteresis loop of the $\mathrm{Fe}_{72} \mathrm{Al}_{14} \mathrm{~B}_{14}$ amorphous ribbon.

In figures 4 and 5 the surface hysteresis loops, measured in the longitudinal direction of the ribbons, are shown. As can be seen, on the wheel surface the saturation field is higher. This result is explained by the general feature of the residual stresses distribution in amorphous ribbons, which consists of a great compressive stress near both surfaces, stronger near the wheel surface, and a weak tensile stress in the central part of the ribbon [13]. Taking into account that in a magnetostrictive material a stress originates a magnetoelastic anisotropy constant given by

$K_{e}=\frac{3}{2} \lambda_{s} \sigma$

the direction of this anisotropy is determined by the sign of $\lambda_{s}$ and $\sigma$. In our case, as the material has a positive magnetostriction and on the surfaces there is a compressive stress, the direction of the magnetoelastic anisotropy results in the longitudinal direction of the ribbon and higher on the wheel than on the air surface.

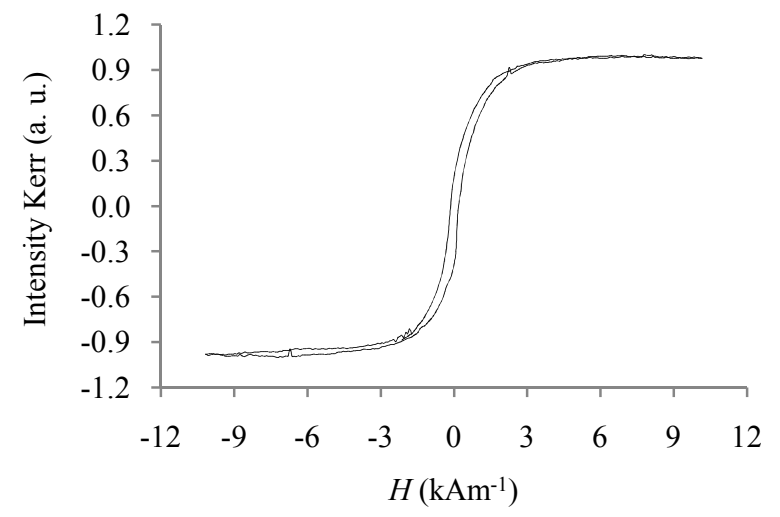

Fig. 4. Air-surface hysteresis loop of the $\mathrm{Fe}_{72} \mathrm{Al}_{14} \mathrm{~B}_{14}$ amorphous ribbon. 


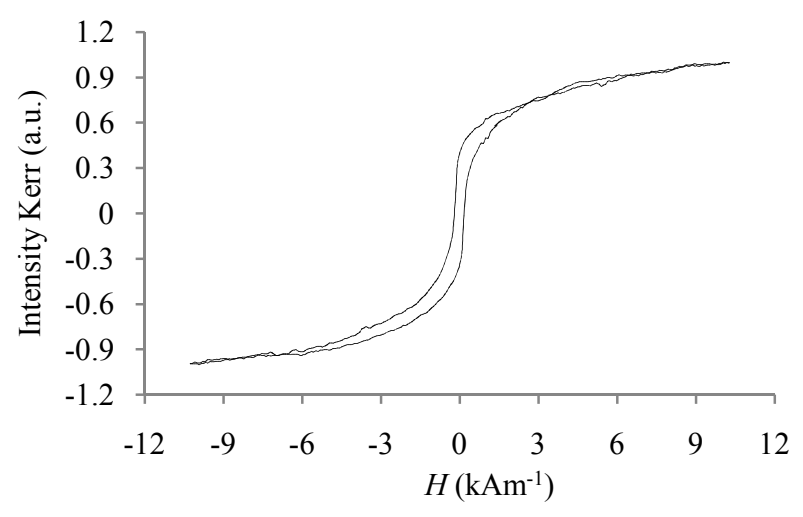

Fig. 5. Wheel surface hysteresis loop of the $\mathrm{Fe}_{72} \mathrm{Al}_{14} \mathrm{~B}_{14}$ amorphous ribbon.

\section{Conclusions}

A new amorphous ribbon obtained by melt spinning is presented together with its magnetic characterization. The material has a saturation magnetization of $1.12 \mathrm{~T}$ and a coercive field of $18 \mathrm{Am}^{-1}$. The ribbon presents a magnetic anisotropy along the longitudinal direction with a value of the anisotropy constant of $1860 \mathrm{Jm}^{-3}$. The wheelsurface has a higher magnetic anisotropy than the airsurface due to the residual stress distribution. The longitudinal magnetostriction $\lambda_{s}$ of the ribbon measured under a transverse magnetic field of $0.9 \mathrm{~T}$ results in $24 \mathrm{ppm}$. The magnetic properties of this amorphous ribbon in combination with this mechanical properties [8], tensile strength of $800 \mathrm{MPa}$ and a Young's modulus of $167 \mathrm{GPa}$, makes this material promising to be used in magnetic sensors.

\section{Acknowledgements}

This work was supported in part by the Spanish Governement under Grant No. MAT2008-06784/MAT.

\section{References}

1. A. García-Arribas, J.M. Barandiarán and J. Gutiérrez, Magnetoelastic Sensors, in: Encyclopedia of Sensors vol. X. Edited by C.A. Grimes, E.C. Dickey and M.V. Pishko. American Scientific Publishers: California, USA 2006, 1-21.

2. F.T. Calkins, A.B. Flatau and M. J. Dapino, J. of Intelligent Mater. Syst. and Struct. 18, 1057 (2007).

3. M. Shimada, J. Appl. Phys. 73, 6872 (1993).

4. I.J. Garshelis and C.A. Jones, J. Appl. Phys. 85, 5468 (1999).

5. H.Y. Liu, L.Q. Yan, J.P. Qu and Y.X. Li, Appl. Phys. Lett. 85, 1751(2004).

6. M.C. Zhang, H.L. Jiang, X.X. Gao, J. Zhu and S.Z. Zhou, J. Appl. Phys. 99, 023903 (2006).

7. R. Ray, V. Panchanthan and B.C. Giessen, US4405368 (2009).

8. J.A. García, J. Carrizo, D. Castrillo, M.A. García, M. Rivas and J. Riba, IWSNCS 10 (2010).
9. J.A. García, A.R. Pierna, J. Carrizo, D. Castrillo, N. Ruíz and F.F. Marzo, IWSNCS 10 (2010).

10. J.A. García and M. Rivas, IEEE Trans. MAG 42, 15 (2006).

11. T. Takahashi, K. Hashimoto, T. Okazaki, Y. Furuya, T. Kubota and Ch. Saito, Script. Mater. 60, 847 (2009).

12. M.P. Ruffoni, S. Pascarelli, R. Grössinger, R. SatoTurtelli, C. Bormio-Nunes and R.F. Pettifer, Phys. Rev. Lett. 101,147202 (2008).

13. M. Tejedor, J.A. García, J. Carrizo and L. Elbaile, J. Mater. Sci. 32, 2337 (1997). 